Method From January 2011 through September 2015, A retrospective study was performed in 27 patients who had hyperacute ischemic stroke and were treated by primary intracranial stenting for refractory re-occlusion during stent-based mechanical thrombectomy. We studied radiologic appearance, clinical presentation and follow up outcomes.

Result Of the 27 patients, 11 patients (40\%) showed radiologic and clinical improvement, 5 patients (18\%) showed clinical improvement with in-stent stenosis, 6 patients $(22 \%)$ had complication with hemorrhagic transformation and 4 patients (14\%) failed recanalization or severe stenosis. In all cases, we use Solitaire FR stent. 6 patients (22\%) were TICI grade III, 10 patients $(37 \%)$ were TICI grade IIb, 4 patients $(14 \%)$ were TICI grade IIa, and 4 patients (14\%) were TICI grade I. After 3 months, 15 patients (55\%) were below mRS 3 points and after 6 month, 18 patients (67\%) were below mRS 3 points.

Conclusions These results indicate that primary intracranial stenting for refractory re-occlusion during stent-based mechanical thrombectomy treatment in acute ischemic stroke is effective technique. Further studies are needed to evaluate longterm occlusion and in-stent stenosis.

Disclosures S. Sheen: None. J. Shin: None.

\section{E-025 FIRST REPORTED CASE OF MECHANICAL THROMBECTOMY FOR ACUTE ISCHEMIC STROKE IN AN INDIVIDUAL WITH A TOTAL ARTIFICIAL HEART}

${ }^{1} \mathrm{M}$ Nezhad, ${ }^{2} \mathrm{P}$ Eboli, ${ }^{3} \mathrm{M}$ Austin, ${ }^{1} \mathrm{~K}$ Schlick, ${ }^{2} \mathrm{M}$ Alexander. ${ }^{1}$ Neurology, Cedars-Sinai Medical Center, Los Angeles, CA; ${ }^{2}$ Neurosurgery, Cedars-Sinai Medical Center, Los Angeles, CA; ${ }^{3}$ Radiology, Cedars-Sinai Medical Center, Los Angeles, CA

\subsection{6/neurintsurg-2016-012589.97}

Background With the results of recent thrombectomy trials, endovascular therapy (EVT) is standard of care in appropriately selected patients with acute ischemic stroke (AIS). We report the first case of mechanical thrombectomy for a patient supported by a total artificial heart (TAH) with AIS.

Clinical Presentation A 37 year old male patient with ischemic cardiomyopathy was found to have right gaze preference, left facial droop, and global aphasia 9 days following implantation of a TAH. Prior to ictus, antithrombotic regimen included daily aspirin and heparin infusion at therapeutic levels. Acute imaging revealed a $6 \mathrm{~mm}$ left M2 thrombus with associated perfusion mismatch seen in the left MCA territory along with a smaller matched perfusion defect seen in the right MCA territory.

Intervention Following confirmation of lesion amenable to mechanical thrombectomy, patient underwent successful TICI 3 revascularization of the left MCA territory with a Solitaire stent retriever. Repeat perfusion imaging revealed resolved left MCA territory mismatch with persistent right MCA matched defect. His speech improved to an expressive aphasia following revascularization.

Conclusion EVT has been proven to be safe and efficacious for AIS. Prior reports have demonstrated benefit for patients with concurrent mechanical circulatory support devices; however, our report is the first case demonstrating efficacy with a $\mathrm{TAH}$ device. Presence of mechanical circulatory support devices should not exclude EVT in individuals with AIS.

Disclosures M. Nezhad: None. P. Eboli: None. M. Austin: None. K. Schlick: None. M. Alexander: None.

\section{E-026 DIAGNOSIS AND MANAGEMENT OF THORACIC AND SHOULDER ARTERIOVENOUS MALFORMATIONS}

W Yakes. Vascular Malformation Center, Englewood, CO

\subsection{6/neurintsurg-2016-012589.98}

Purpose To determine the efficacy of Endovascular Repair of Thoracic and Shoulder Arteriovenous Malformations (AVMs). Previous reports have documented the futility of nBCA and amputation in treating these lesions in this specific anatomy. Materials and methods Twelve patients ( 8 female, 4 male) presented for repair of shoulder and thoracic AVMs. Three patients had extension of AVM to the supraclavicular and axillary areas. Two patients had multiple AVMs. Seven patients had previous failed therapies (embo: PVA/coils/gelfoam; surgeries: excisions/arterial bypass). All patients underwent ethanol endovascular AVM repair; four patients had additional coil embolizations (132 treatments). Patient age range 18-76 years; mean age 36 .

Results Eleven patients are cured at long-term arteriographic follow-up (follow-up 22-192 months; mean follow-up: 42 months). One patient with bilateral shoulder AVM and multiple other AVMs therapy is on-going. Complications include two patients with minor superficial blisters, one patient with transient left radial nerve injury with complete recovery and one patient with clot embolus to hand, $\mathrm{Rx}$ with urokinase w/ distal $3^{\text {rd }}$ phalanx removed. Thus, major complications were 2/132 procedures, one being transient.

Conclusions A report of shoulder AVM repair in JVIR documented failure of nBCA approach even coupled with quadrant amputation whereby recurrence was universal. These authors stated that shoulder AVMs were not possible to treat. This report documents that cure of these difficult lesions is possible with ethanol endovascular approaches and direct puncture approaches. No other publications in world literature documents cure of AVMs in this anatomy.

Long-term cures are noted with the use of ethanol, and ethanol and coils to successfully treat these complex, problematic lesions. A low major complication rate is noted. This patient series finally documents a curative procedure for this daunting lesion.

Disclosures W. Yakes: None.

\section{E-027 MECHANICAL THROMBECTOMY FOR ACUTE ISCHEMIC STROKE IN POST-SURGICAL PATIENTS}

${ }^{1}$ M Litao, ${ }^{1} \mathrm{H}$ Bhamra, ${ }^{2} \mathrm{~K}$ DeSousa, ${ }^{2} \mathrm{E}$ Raz, ${ }^{3} \mathrm{E}$ Nossek, ${ }^{1} \mathrm{~A}$ Favate, ${ }^{4} \mathrm{M}$ Shapiro, ${ }^{5} \mathrm{~T}$ Becske, ${ }^{2} \mathrm{P}$ Nelson. 'Neurology, New York University, New York, NY; ${ }^{2}$ Radiology, New York University, New York, NY; ${ }^{3}$ Neurosurgery, Maimonides Medical Center, New York, NY; ${ }^{4}$ Neurology/Radiology, New York University, New York, NY; ${ }^{5}$ Neurology, Rochester Regional Health, Rochester, NY

\subsection{6/neurintsurg-2016-012589.99}

Introduction Ischemic strokes in the post-surgical period may negatively impact clinical outcome. Major surgery within the previous 14 days is a relative contraindication to intravenoustPA administration. Mechanical thrombectomy may thus become a valid treatment option. We present our experience in a series of patients who presented with a clinical stroke syndrome in the post-surgical period who underwent emergent angiography with intent to treat.

Methods This is a retrospective review of patients who were treated from January 2012 to March 2015 in our institution. 
Patients who had emergent angiography after presenting with clinical stroke during the post-surgical period were included. Type of surgery, clinical characteristics, imaging studies, treatment variables, and discharge modified Rankin scores were collected.

Results 15 patients had emergent angiography in the post-surgical period. The mean age is 68.87 years. $87 \%(13 / 15)$ had cardiovascular surgery, $8(62 \%)$ of which were valvular repairs. $57 \%(8 / 14)$ presented in the immediate post-surgical period. The mean NIHSS was 18. Mean symptom duration from time last known well was 256 minutes. 10/11 (91\%) had perfusion deficits on CTP.

$60 \%(9 / 15)$ had proximal vessel occlusion while $33 \%$ $(5 / 15)$ had distal occlusions, and 1 had no occlusion. Of the 9 patients with proximal vessel occlusions, 7 underwent thrombectomy with the Solitaire device achieving a 57\% (4/7) TICI $2 \mathrm{~b} / 3$ recanalization rate. The mean mRS for all patients in the series is 3.79. Patients with TICI $2 \mathrm{~b} / 3$ recanalization did significantly better at discharge than those who did not, mean mRS of 1.67 vs. $5, \mathrm{p}=0.019$.

Conclusion In our experience, emergent angiography among post-surgical stroke patients showed proximal vessel occlusion in more than half of patients, making them potential candidates for mechanical thrombectomy. Those who achieved TICI $2 \mathrm{~b} / 3$ recanalization performed clinically better than those who did not. This may indicate the potential benefit of mechanical thrombectomy among post-surgical stroke patients. The overall lower recanalization rates in this cohort may reflect atypical clot characteristics encountered in post-surgical patients and would be an interesting avenue for further investigation.

Disclosures M. Litao: None. H. Bhamra: None. K. DeSousa: None. E. Raz: None. E. Nossek: None. A. Favate: None. M. Shapiro: 2; C; Covidien. T. Becske: 2; C; Covidien. P. Nelson: 2; C; Covidien.

\section{E-028 MANAGEMENT OF TONGUE VENOUS AND LYMPHATIC MALFORMATIONS}

W Yakes. Vascular Malformation Center, Englewood, CO

\subsection{6/neurintsurg-2016-012589.100}

Purpose To determine the efficacy of ethanol embolization in management of tongue venous and lymphatic malformations. Materials and methods Thirty-nine patients (22 females, 17 males; mean age: 38 years) presented with tongue low-flow malformations. Forty-seven patients had undergone 61 failed previous procedures (embo, laser, surgery, steroid injection, alpha-interpheron, radiation). All patients had baseline arteriograms and MRs. All patients underwent direct puncture ethanol endovascular therapy.

Results Of 39 patients with venous and lymphatic malformations, 32 patients had dramatic reduction and 7 patients' therapy is on-going with concurrent reductions (mean f/up: 60 months). One patient with AVM required additional surgery and 1 patient with mixed veno-lymphatic malformation required surgical debulking of excess tissues. Minor complications such as tongue blisters ( 9 instances) healed spontaneously; 3 tongue focal necrosis injuries healed spontaneously; 3 infections responded to antibiotic treatment; 1 focal tongue hemi numbness resolved.
Conclusion Ethanol embolotherapy is a primary and consistent form of therapy to eradicate low-flow vascular malformations of the tongue permanently at long-term follow-up. Rarely is concurrent surgery required. Ethanol sclerotherapy is a curative treatment in which recurrences do not occur and permanent ablations are the rule. Complications that occur are minor and rare.

Disclosures W. Yakes: None.

\section{E-029 MECHANICAL THROMBECTOMY IN PEDIATRIC ACUTE ISCHEMIC STROKE: CLINICAL OUTCOMES AND LITERATURE REVIEW}

${ }^{1} \mathrm{~T}$ Madaelil, ${ }^{1} \mathrm{~A}$ Kansagra, ${ }^{2} \mathrm{C}$ Derdeyn, ${ }^{1} \mathrm{D}$ Cross, ${ }^{1} \mathrm{C}$ Moran. ${ }^{1}$ Neuroradiology, Mallinckrodt Institute of Radiology, Saint Louis, MO; ${ }^{2}$ Neuroradiology, Neurology, Neurosurgery, University of lowa Hospitals and Clinics, lowa City, IA

\subsection{6/neurintsurg-2016-012589.101}

There is limited data on outcomes of mechanical thrombectomy for pediatric stroke using modern devices. In this study, we report two cases of pediatric acute ischemic stroke treated with mechanical thrombectomy, both with good angiographic result (TICI 3) and clinical outcome (no neurological deficits at 90 days). In addition, we conducted a literature review of all previously reported cases describing the use of modern thrombectomy devices. Including our two cases, the aggregate rate of partial or complete vessel recanalization was $100 \%$ $(22 / 22)$, and the aggregate rate of favorable clinical outcome was 91\% (20/22). This preliminary evidence suggests that mechanical thrombectomy with modern devices may be a safe and effective treatment option in pediatric patients with acute ischemic stroke.

Disclosures T. Madaelil: None. A. Kansagra: None. C. Derdeyn: None. D. Cross: None. C. Moran: 2; C; Medtronic Neurovascular.

\section{E-030 TRANSVENOUS MICROGUIDEWIRE LOOPING TECHNIQUE FOR BREACH OF IPSILATERAL INFERIOR PETROSAL SINUS OCCLUSIONS EN ROUTE TO CAVERNOUS SINUS DURAL ARTERIOVENOUS FISTULAS}

Y Cho, H Kang, M Han. Radiology, Seoul National University Hospital, Seoul, Republic of Korea

10.1136/neurintsurg-2016-012589.102

Purpose Transarterial access to dural arteriovenous fistulas (dAVFs) has been popularized by device improvements and novel embolic materials. However, this approach is limited in the cavernous sinus (CS) due to related complications and low cure rates. Although a transvenous approach, via ipsilateral inferior petrosal sinus (IPS), may be more suitable for CSdAVFs, microcatheter delivery is occasionally impeded by ipsilateral IPS occlusion. Described herein is a novel microguidewire looping method to breach such occlusions, thus enabling access to CS lesions.

Methods A microcatheter is initially advanced into IPS orifice, and a microguidewire is passed into occluded IPS. Looping is easily achieved through the resistance met. With greater support of the guiding catheter, the microguidewire (still looped) is then advanced into CS. When nearing CS, the 